\title{
Gli anticoagulanti orali non vitamina K-dipendenti nei pazienti con fibrillazione atriale non valvolare ed end-stage renal disease (ESRD): un matrimonio impossibile?
} \author{
Francesca Santoboni ${ }^{1}$, Antonio De Pascalis ${ }^{7}$, Claudio Ronco ${ }^{8}$ \\ ${ }^{1}$ UOC Nefrologia e Dialisi, Ospedale "L. Parodi-Delfino", Colleferro (RM) \\ ${ }^{2}$ UOC Nefrologia e Dialisi, Ospedale "S. Paolo", Civitavecchia (RM) \\ ${ }^{3}$ Divisione di Nefrologia, Ospedale "S. Gerardo", Monza \\ ${ }^{4}$ UOC Nefrologia e Dialisi, ASTT Lariana, Ospedale "S. Anna", Como \\ ${ }^{5}$ Dipartimento di Scienze della Salute, Divisione di Nefrologia, Ospedale "S. Paolo", Milano \\ ${ }^{6}$ Nefrologia e Dialisi, USL Umbria 1, Todi (PG) \\ 'UOC Nefrologia e Dialisi, Ospedale "V. Fazzi", Lecce \\ ${ }^{8}$ International Renal Research Institute, Ospedale "S. Bortolo", Vicenza
}

Luca Di Lullo ${ }^{1}$, Fulvio Floccari ${ }^{2}$, Rodolfo Rivera ${ }^{3}$, Vincenzo Barbera ${ }^{1}$, Antonio Bellasi ${ }^{4}$, Mario Cozzolino ${ }^{5}$, Antonio Selvi ${ }^{6}$,

\begin{abstract}
Non-vitamin-K-dependent oral anticoagulants in end-stage renal disease patients with non-valvular atrial fibrillation: an impossible wedding?

Atrial fibrillation (AF) is the most common arrhythmia in patients with chronic kidney disease (CKD). In this population, AF is associated with an increased risk of thromboembolism and stroke as a result of a progressive decline in the glomerular filtration rate. However, CKD patients, in particular those on renal replacement therapy, also have an increased risk of bleeding, especially from the gastrointestinal tract. Oral anticoagulation is the most effective form of thromboprophylaxis in patients with AF presenting an increased risk of stroke. Limited evidence of its efficacy, the increased risk of bleeding as well as some concern regarding the use of warfarin in CKD have often resulted in the underuse of anticoagulation in CKD patients. A large body of evidence suggests that non-vitamin-K-dependent oral anticoagulants (NOACs) significantly reduce the risk of stroke, intracranial hemorrhage and mortality. Hence, they are currently recommended for patients with AF at risk of stroke. However, the metabolism of NOACs is largely dependent on the kidneys for elimination and little is known about their use in patients with creatinine clearance $<25 \mathrm{~mL} / \mathrm{min}$, who have been excluded from all pivotal phase III NOAC trials. This review focuses on the current pharmacokinetic, observational and prospective data on NOACs in patients affected by moderate to advanced CKD (creatinine clearance $15-49 \mathrm{~mL} / \mathrm{min}$ ) and in those on dialysis.
\end{abstract}

Keywords: Anticoagulation therapy, Atrial fibrillation, Chronic kidney disease, Non-vitamin-K oral anticoagulants (NOACs), Warfarin

Accepted: April 18, 2017

Published online: May 17, 2017

Indirizzo per la corrispondenza:

Dr. Luca Di Lullo

UOC Nefrologia e Dialisi

Ospedale L. Parodi-Delfino

Piazza Aldo Moro 1

00034 Colleferro (RM)

dilulloluca69@gmail.com 


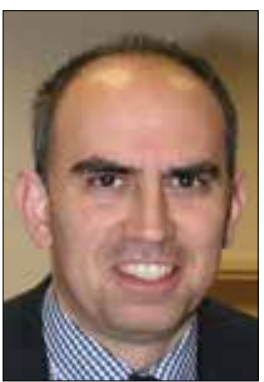

\section{Fibrillazione atriale e malattia renale cronica: epidemiologia e fattori di rischio}

La fibrillazione atriale (FA) rappresenta la forma più comune di aritmia cardiaca e ha una prevalenza pari all'1$2 \%$ della popolazione generale $(1,2)$. Tale percentuale cresce progressivamente con il progredire dell'età anagrafica arrivando al $14 \%$ circa nei pazienti con età superiore agli 80 anni (3).

La FA si configura, inoltre, come fattore di rischio per morbidità e mortalità cardiovascolari (con circa 112.000 decessi nel 2013 a fronte dei 90.000 del 1990) grazie anche all'aumentata incidenza di complicanze tromboemboliche (incluso l'ictus ischemico) (4).

È altrettanto noto come la presenza di malattia renale cronica (Chronic Kidney Disease, CKD) comporti un aumento del rischio di morbidità e mortalità cardiovascolari, infatti circa il 60-70\% dei decessi dei pazienti affetti da malattia renale cronica, soprattutto terminale (End-Stage Renal Disease, ESRD), è dovuto a cause cardiovascolari (5).

Lo stretto legame tra FA e CKD è testimoniato dal fatto che la presenza di un deficit della funzione renale determina non solo alterazioni morfo-funzionali a livello dei cardiomiociti, ma anche modificazioni del quadro emopoietico e della risposta infiammatoria, tutti fattori in grado di incidere sull'insorgenza di FA (6).

Nei pazienti affetti da CKD, la percentuale di popolazione affetta da FA oscilla dal $19 \%$ al $24 \%$ con un picco pari a circa il $27 \%$ in coloro i quali sono affetti da ESRD (7); del resto, già nello studio di Framingham, la prevalenza di FA nei pazienti affetti da malattia renale raggiungeva livelli pari a 15 volte quelli della popolazione generale (8).

Nello studio CRIC (Chronic Renal Insufficiency Cohort), la FA era presente nel $18 \%$ degli oltre 3000 pazienti affetti da CKD ed era associata alla presenza di fattori di rischio cardiovascolare tradizionali quali età avanzata, sesso femminile, assunzione di nicotina e storia di scompenso cardiaco.

In base ai dati dello studio ARIC (Atherosclerosis Risk in Communities), il rischio relativo di sviluppare FA era di 3.2 volte maggiore nella popolazione con CKD a uno stadio 4-5 rispetto ai soggetti con funzione renale conservata (9-11).

Nei pazienti affetti da CKD allo stadio 3-5, inoltre, è stata documentata un'associazione statisticamente significativa tra rischio di sviluppo di FA e livelli di albuminuria (11), con un aumento del rischio di sviluppare FA pari a circa il doppio nei pazienti microalbuminurici (10).

Nei pazienti affetti da ESRD, la prevalenza di FA varia tra il $7 \%$ e il $27 \%(8,12,13)$ con un'incidenza stimata tra 3.1 e 5.9 per 100 pazienti/anno (14-16). Uno studio di coorte comprendente circa 260.000 pazienti di età superiore ai 65 anni ha permesso di individuare un'incidenza di FA pari a 148 casi per 1000 pazienti/anno, con un incremento dell'incidenza stessa pari all'11\% in un arco temporale di 12 anni (17).

È, ormai, ben documentato come lo stadio terminale della malattia renale cronica sia associato a diverse co-morbidità di natura cardiovascolare e ad alcuni fattori di rischio in grado di indurre l'insorgenza di FA quali età avanzata, ipertensione arteriosa, malattia coronarica, ipertrofia ventricolare sinistra e insufficienza cardiaca congestizia (18).

La stessa malattia renale cronica terminale e l'inizio del trattamento sostitutivo emodialitico sono in grado di apportare alterazioni di tipo emodinamico e metabolico tali da contribuire alla genesi della FA, in particolar modo nel periodo interdialitico (19).

Tra i fattori di rischio peculiari della CKD che mostrano un significativo grado di associazione con la presenza di FA deve essere menzionata l'anemia, come evidenziato dallo studio di popolazione condotto nella prefettura giapponese di lbaraki comprendente circa 130.000 pazienti. Nella popolazione studiata veniva, infatti, documentata una maggiore incidenza di FA nei pazienti anemici, soprattutto, in coloro nei quali l'anemia era associata alla presenza di malattia renale (20). Nei pazienti affetti da CKD allo stadio 4-5, la correzione dei livelli di emoglobina mediante terapia con eritropoietina non solo era in grado di ridurre il grado di ipertrofia ventricolare sinistra e l'incidenza di eventi cardiovascolari, ma anche di ridurre l'incidenza di FA (21).

Anche l'associazione di FA con il rischio di stroke è un punto da considerare, poiché l'incidenza di quest'ultimo risulta aumentata di circa 5 volte rispetto alla popolazione di soggetti esenti da FA (1) e i pazienti affetti da CKD e da FA presentano un rischio più elevato di stroke ischemico rispetto ai soggetti con funzione renale conservata $(22,23)$. Livelli particolarmente ridotti di filtrato glomerulare sono significativamente associati a un incremento del rischio di stroke ischemico e ad altre patologie sistemiche su base tromboembolica, indipendentemente dagli altri fattori di rischio presenti nei pazienti affetti da FA (24). Inoltre, il rischio di sviluppare stroke è decisamente più elevato nei pazienti affetti da CKD indipendentemente dal grado di $\mathrm{CHA}_{2} \mathrm{DS}_{2}$-VASc score, un indice routinariamente utilizzato nella valutazione del rischio tromboembolico.

Dati del registro nazionale danese comprendente 132.000 pazienti affetti da CKD e FA evidenziano come il rischio di stroke aumenti di circa due volte nei pazienti sottoposti a trattamento emodialitico o sottoposti a trapianto renale (25).

Altri dati concordanti sono quelli di Vazquez et al., i quali hanno evidenziato come la presenza di FA sia associata, nei pazienti in trattamento emodialitico, a un rischio di stroke superiore di circa 10 volte rispetto ai pazienti con normale ritmo sinusale all'esame elettrocardiografico (14).

Fanno da contraltare altri studi clinici che, pur evidenziando un maggior rischio cardiovascolare nei pazienti affetti da CKD e FA, non documentano un significativo aumento dell'incidenza di stroke $(26,27)$. 
Un ultimo punto che va preso in considerazione prima di passare al capitolo riguardante gli approcci terapeutici nel paziente affetto da CKD e FA è quello riguardante le alterazioni del sistema della coagulazione.

Nei pazienti affetti da CKD, il progressivo danneggiamento dell'apparato endoteliale e del sistema vascolare capillare della midollare renale è, verosimilmente, alla base della progressione del danno, come evidenziato dall'aumento degli indici infiammatori (28).

A quanto descritto sopra, si devono aggiungere le alterazioni dei sistemi enzimatici coinvolti nei processi di emostasi, con un conseguente incremento dell'incidenza di fenomeni emorragici e tromboembolici (29).

Recenti dati della letteratura evidenziano, infine, un incremento dei fenomeni tromboembolici, probabilmente correlati a uno stato di ipercoagulabilità, a sua volta imputabile al grado di ipoalbuminemia spesso presente nei pazienti con CKD.

Sia la patologia su base emorragica che quella a genesi tromboembolica possono avere importanti e profonde ripercussioni nel paziente con CKD e FA e richiedere l'impiego di una terapia anticoagulante (30).

\section{La terapia anticoagulante}

La prescrizione di una terapia anticoagulante rappresenta una pietra miliare nel trattamento del paziente affetto da FA, allo scopo di ridurre quanto più possibile il rischio di eventi cerebrovascolari acuti correlati alla presenza della stessa FA. Allo stesso tempo, è ben documentato come l'impiego di una terapia anticoagulante esponga il paziente a un maggior rischio di sanguinamento (31).

Facendo riferimento alle Linee Guida emanate dalla European Society of Cardiology (ESC), è obbligatorio fare riferimento, rispettivamente, agli score $\mathrm{CHA}_{2} \mathrm{DS}_{2}$ VASc e HAS-BLED atti a quantificare il rischio di stroke e di sanguinamento nei pazienti affetti da FA (1).

Alla luce delle suddette Linee Guida, il trattamento anticoagulante è raccomandato nei pazienti affetti da FA con rischio elevato di stroke ( $\mathrm{CHA}_{2} \mathrm{DS}_{2} \mathrm{VASc} \geq 2$ ) e andrebbe preso in considerazione nei pazienti con uno score comunque maggiore di 1.

Sempre in base alle Linee Guida, diverse opzioni terapeutiche sono tuttora disponibili, a partire dagli antagonisti della vitamina K (VKA), come il warfarin in grado di bloccare la sintesi di diversi fattori della coagulazione vitamina K-dipendenti.

Di recente introduzione sul mercato sono i cosiddetti anticoagulanti orali non vitamina K-dipendenti (NOAC), quali l'inibitore diretto della trombina (dabigatran) e gli inibitori diretti del fattore $X$ attivato $(X a)$ : rivaroxaban, apixaban ed edoxaban.

Nei trial clinici di registrazione, tutti i NOAC hanno evidenziato una non-inferiorità nei confronti del warfarin in termini di efficacia, mostrando, al contempo, di dare luogo a una minore incidenza di emorragie intracraniche rispetto al warfarin stesso (32).

Tutte le molecole appartenenti alla famiglia dei NOAC presentano un grado più o meno importante di eliminazio- ne renale a partire dall' $80 \%$ di dabigatran, fino ad arrivare al $50 \%$ di edoxaban, al $33 \%$ di rivaroxaban e al $27 \%$ di apixaban.

II trattamento con NOAC in pazienti con valori di eGFR $<30 \mathrm{~mL} / \mathrm{min} / 1.73 \mathrm{~m}^{2}$, con l'eccezione del dabigatran, è stato approvato in Europa, ma non viene, tuttora, raccomandato in quanto risultano ancora deficitari i dati riguardanti la tollerabilità degli stessi in questa popolazione di pazienti (1).

Per tale motivo, durante la terapia con NOAC la funzione renale dopo una valutazione preliminare andrebbe attentamente monitorata, specialmente nei pazienti anziani, in chi presenta un maggior rischio di ipovolemia e/o disidratazione e in chi assume una terapia polifarmacologica.

La presenza di una condizione di malattia renale cronica impone la riduzione dei dosaggi di NOAC: per esempio, il dabigatran andrebbe assunto a un dosaggio di $110 \mathrm{mg}$ BID, il rivaroxaban di $15 \mathrm{mg}$ OD, l'apixaban di $2.5 \mathrm{mg}$ BID (in presenza di 2 dei tre seguenti fattori: età $\geq 80$ anni, peso corporeo $\leq 60 \mathrm{~kg}$ e creatinina sierica $\geq 1.5 \mathrm{mg} / \mathrm{dL}$ ) e l'edoxaban di $30 \mathrm{mg}$ OD (1).

Per quanto concerne l'impiego del dabigatran, per i pazienti con compromissione severa della funzione renale (eGFR $<15 \mathrm{~mL} / \mathrm{min}$ ) la Food and Drug Administration (FDA) ha approvato, solo negli USA, il dosaggio di $75 \mathrm{mg}$ BID.

Comunque, nonostante l'ampio ventaglio di soluzioni alternative, allo stato attuale la terapia con warfarin è ancora quella più utilizzata, anche se non è scevra da complicanze, in particolar modo proprio nei pazienti affetti da CKD. Infatti il warfarin è coinvolto nei processi che riguardano i meccanismi fisiopatologici in grado di favorire la formazione di calcificazioni vascolari (inibizione della Gla protein) e gioca un ruolo di primo piano nei processi di natura apoptotica che interessano le cellule endoteliali renali (33). Il warfarin è risultato, inoltre, il responsabile di una vera e propria nefropatia da anticoagulante in pazienti con funzione renale sia conservata che ridotta (34).

Alla luce di queste evidenze, laddove sia possibile, le ultime Linee Guida ESC incoraggiano e consigliano l'impiego in prima battuta dei NOAC anche in pazienti con CKD allo stadio 3-4 (con eGFR compreso tra 15 e $49 \mathrm{~mL} / \mathrm{min} / 1.73 \mathrm{~m}^{2}$ ).

\section{Gli anticoagulanti orali non vitamina K-dipendenti (NOAC)}

La Tabella I e le Figure 1 e 2 evidenziano le caratteristiche farmacologiche dei 4 NOAC confrontati con il warfarin. Di seguito vengono riportate le principali informazioni riguardanti le nuove molecole anticoagulanti.

\section{Dabigatran}

Dabigatran è stato il primo NOAC a essere introdotto nella pratica clinica ed è, dal punto di vista farmacologico, un inibitore diretto della trombina, approvato dalla FDA al do- 
TABELLA I - Caratteristiche farmacologiche del warfarin e degli anticoagulanti orali non vitamina-K dipendenti (NOAC)

\begin{tabular}{|c|c|c|c|c|c|}
\hline Parametri & Warfarin & Dabigatran & Apixaban & Rivaroxaban & Edoxaban \\
\hline Volume di distribuzione (litri) & 8 & $50-10$ & 2 & 50 & 107 \\
\hline Legame proteico (\%) & 99 & 35 & 87 & 95 & 54 \\
\hline Emivita (ore) & $20-60$ & $7-17$ & $8-15$ & $7-13$ & $9-11$ \\
\hline $\begin{array}{l}\text { Livello inferiore di eGFR previsto dalla } \\
\text { FDA per l'impiego }\end{array}$ & $\begin{array}{l}\text { Può essere utilizzato nei pa- } \\
\text { zienti in trattamento dialitico }\end{array}$ & 15 & $\begin{array}{c}<15 \\
\text { (solo negli USA) }\end{array}$ & 15 & 15 \\
\hline
\end{tabular}

$\mathrm{CrCl}=$ creatinina clearance $; \mathrm{FDA}=$ Food and drug administration; FFP = plasma fresco congelato; 4F-PPC = complesso concentrato fattore 4-protrombina (4-factor prothrombina); $\mathrm{HR}=$ hazard ratio.

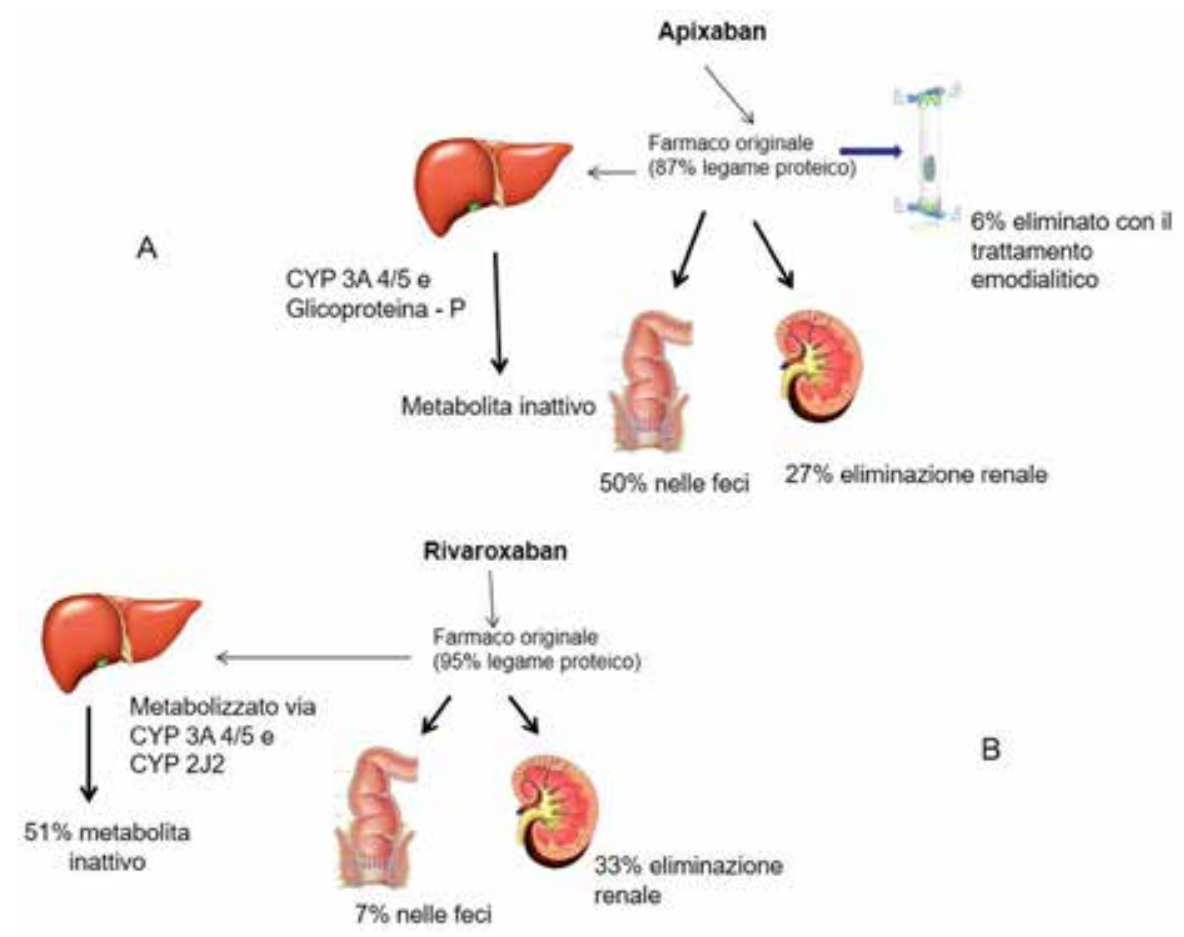

Fig. 1 - Profilo farmacologico di Apixaban (A) e Rivaroxaban (B).

saggio di $150 \mathrm{mg}$ BID per pazienti con eGFR $>50 \mathrm{~mL} / \mathrm{min}$ e di $110 \mathrm{mg}$ BID per soggetti con eGFR compreso tra 30 e 50 $\mathrm{mL} / \mathrm{min}$. II dabigatran presenta un metabolismo renale pari all' $80 \%$ e il grado di compromissione della funzione renale gioca un ruolo fondamentale nella clearance stessa del farmaco. Nello studio RE-LY (Randomized Evaluation of LongTerm Anticoagulation Therapy), il dabigatran si è dimostrato superiore al warfarin nella prevenzione dello stroke e della patologia tromboembolica in pazienti affetti da FA (35). Alla luce del suo profilo farmacologico, le Linee Guida EHRA (European Heart Rhythm Association) ed ESC ne sconsigliano I'impiego in pazienti con eGFR $<30 \mathrm{~mL} / \mathrm{min}$ (36). Si tratta, nell'ambito dei NOAC, dell'unica molecola rimovibile me- diante trattamento emodialitico, anche se ci si può aspettare una sorta di "rebound" nei livelli plasmatici post-dialisi dovuto all'elevato volume di distribuzione del farmaco (37).

Solo per il territorio USA, la FDA ha approvato il dosaggio di $75 \mathrm{mg}$ BID nei pazienti con eGFR compreso tra $15 \mathrm{e}$ $30 \mathrm{~mL} / \mathrm{min}$ (36).

\section{Rivaroxaban}

Rivaroxaban è un inibitore diretto del fattore Xa con eliminazione renale pari al 33\% (38). Nello studio ROCKET$\mathrm{AF}$, trial randomizzato controllato condotto su oltre 14.000 pazienti a rischio moderato-severo per stroke e con valori 


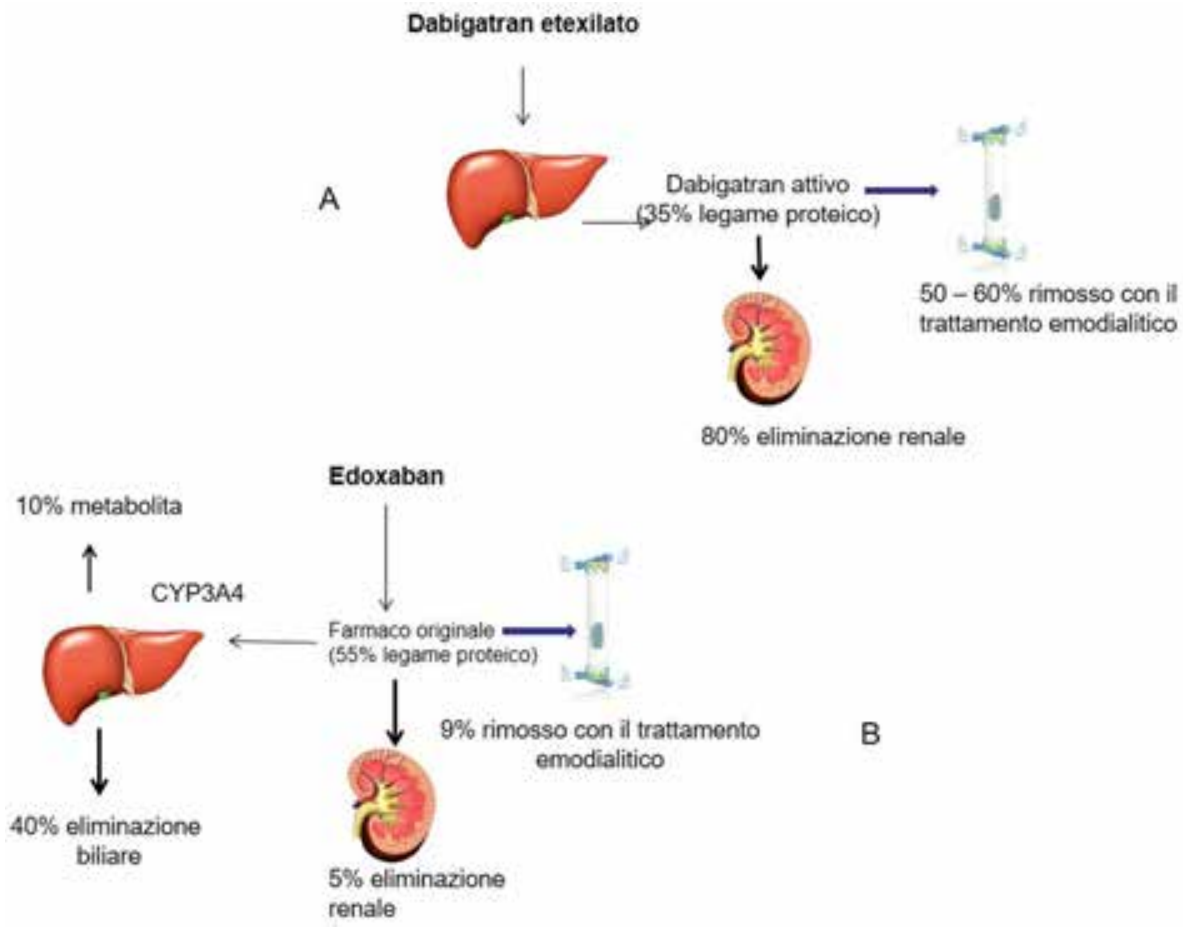

Fig. 2 - Profilo farmacologico di Dabiga$\operatorname{tran}(\mathbf{A})$ ed Edoxaban (B).

di eGFR >30 mL/min, il rivaroxaban (al dosaggio di $20 \mathrm{mg}$ / die) ha evidenziato una non inferiorità rispetto al warfarin nella prevenzione dello stroke e dell'embolismo sistemico e un rischio di sanguinamento maggiore sostanzialmente equivalente (39).

Circa 3000 pazienti (pari al $20.7 \%$ dell'intera popolazione in studio) con valori di eGFR compresi tra 30 e $49 \mathrm{~mL} / \mathrm{min}$ sono stati trattati con una dose di $15 \mathrm{mg} /$ die; questi pazienti hanno presentato degli outcome assolutamente sovrapponibili a quelli dei pazienti con normofunzione renale e trattati con il dosaggio di $20 \mathrm{mg} /$ die (40).

Rivaroxaban è stato approvato dalla FDA nel mese di novembre 2011 e le Linee Guida EHRA (2015) ed ESC (2016) ne raccomandano il dosaggio di $20 \mathrm{mg} /$ die nei pazienti con eGFR $\geq 50 \mathrm{~mL} / \mathrm{min}$ e di $15 \mathrm{mg} /$ die in coloro i quali presentano un valore di eGFR compreso tra 15 e $49 \mathrm{~mL} / \mathrm{min}$. Dati risultanti dall'impiego del dosaggio ridotto non hanno evidenziato né un peggioramento degli outcome né tantomeno un aumento del rischio emorragico nei confronti della stessa tipologia di pazienti trattati con warfarin (38).

Allo stato attuale rivaroxaban non è raccomandato nei pazienti affetti da ESRD (eGFR $<15 \mathrm{~mL} / \mathrm{min}$ ) o sottoposti a trattamento emodialitico, dal momento che, anche in questo caso, vi è una rimozione piuttosto modesta della molecola attraverso le membrane di dialisi (41).

Le ultime evidenze della letteratura testimoniano, però, che il rivaroxaban possa cominciare a essere preso in considerazione anche nei pazienti con ESRD o sottoposti a trattamento emodialitico.

\section{Apixaban}

Apixaban è un inibitore diretto del fattore Xa e presenta una clearance renale pari al 27\%. Nel trial AVERROES (5599 pazienti con creatinina sierica $<2.5 \mathrm{mg} / \mathrm{dL}$ ) è stata evidenziata la superiorità di apixaban nei confronti dell'acido acetilsalicilico per quanto riguarda la prevenzione sia dello stroke che dell'embolizzazione sistemica. I pazienti arruolati erano tutti soggetti affetti da FA non valvolare per i quali non era proponibile il trattamento con warfarin e che venivano randomizzati ad assumere aspirina o apixaban al dosaggio di $5 \mathrm{mg}$ BID o $2.5 \mathrm{mg}$ BID in presenza di 2 dei seguenti 3 criteri: creatininemia compresa tra 1.5 e $2.5 \mathrm{mg} / \mathrm{dL}$, età $>80$ anni e peso corporeo $<60 \mathrm{~kg}$.

I risultati del trial mostrano inoltre l'assenza di significative differenze in termini di rischio di sanguinamento maggiore (42).

Nel trial ARISTOTLE (Apixaban for Reduction in Stroke and Other Thromboembolic Events in Atrial Fibrillation), nel quale sono stati arruolati 18.201 soggetti con livelli di creatinina sierica $<2.5 \mathrm{mg} / \mathrm{dL}$, i pazienti sono stati randomizzati a ricevere apixaban $5 \mathrm{mg}$ BID o warfarin; in una ridotta percentuale di pazienti (circa il $4 \%$ ), apixaban è stato somministrato al dosaggio di $2.5 \mathrm{mg}$ BID utilizzando gli stessi criteri dello studio AVERROES.

Apixaban è stato approvato dalla FDA nel dicembre 2012 per la prevenzione dello stroke e del tromboembolismo in pazienti con FA non valvolare al dosaggio di $5 \mathrm{mg}$ BID nei pazienti con eGFR $\geq 50 \mathrm{~mL} / \mathrm{min}$ e di $2.5 \mathrm{mg}$ BID in presenza di due dei seguenti criteri: creatininemia $>1.5 \mathrm{mg}$, età superiore a 80 anni e peso corporeo $<60 \mathrm{mg}(43)$. 
Apixaban non è dializzabile, se non in minima percentuale (6-7\%) e non è, pertanto, indicato in pazienti sottoposti a trattamento sostitutivo anche se, come verrà evidenziato nella sezione dedicata, iniziano a essere pubblicati i primi dati su pazienti con ESRD e la FDA ha posto l'indicazione all'impiego nei pazienti con eGFR $<15 \mathrm{~mL} / \mathrm{min}$.

\section{Edoxaban}

Edoxaban, in ordine cronologico, è l'ultimo NOAC inibitore diretto del fattore Xa apparso sul mercato e presenta un metabolismo renale pari a circa il 50\%. L'esposizione al farmaco è risultata incrementata del $32 \%$, del $74 \%$ e del $72 \%$ nei pazienti con una compromissione della funzione renale rispettivamente lieve, moderata o severa (44). II trial ENGAGE-AF TIMI ha arruolato circa 21.000 pazienti affetti da FA ed eGFR $>30 \mathrm{~mL} / \mathrm{min}$, evidenziando una non inferiorità di edoxaban al dosaggio di $60 \mathrm{mg} /$ die (30 mg/die in pazienti con eGFR compreso tra 30 e $49 \mathrm{~mL} / \mathrm{min}$ e peso corporeo $<60 \mathrm{~kg}$ ) rispetto al trattamento con warfarin nella prevenzione di stroke e tromboembolismo (45). Lo stesso trial ha, inoltre, evidenziato un rischio decisamente inferiore di sanguinamento maggiore nei pazienti trattati con edoxaban $(39,46)$.

Uno studio prospettico della durata di tre mesi condotto su 93 pazienti trattati con un dosaggio di edoxaban pari a $15 \mathrm{mg} /$ die e affetti da CKD allo stadio 4 ha documentato un rischio di sanguinamento sovrapponibile a quello presentato da pazienti trattati con un dosaggio di 30 o $60 \mathrm{mg} /$ die (47).

Anche per edoxaban cominciano ad apparire in letteratura i primi studi condotti su pazienti affetti da ESRD ma, considerata la ridotta capacità di eliminazione del farmaco (9\%) mediante trattamento emodialitico, al momento il suo impiego è controindicato nei pazienti sottoposti a tale trattamento (48).

A causa dell'incremento del rischio di stroke ischemico rispetto al warfarin nei pazienti con eGFR >95 mL/min, la FDA ha controindicato l'impiego di edoxaban in questa popolazione di pazienti anche se, allo stato attuale, non sono state trovate spiegazioni plausibili per tali evidenze cliniche (49).

\section{Il trattamento anticoagulante nei pazienti con ESRD}

Nei pazienti con ESRD, la presenza di disfunzione endoteliale, aterosclerosi, infiammazione cronica e comorbidità cardiovascolari rappresenta un'evidenza clinica ormai consolidata e solo parzialmente corretta dall'inizio del trattamento sostitutivo della funzione renale.

I pazienti affetti da ESRD presentano una maggiore incidenza di stroke ed emorragie e una particolare suscettibilità a fenomeni di natura emorragica, attribuibile a difetti di tipo quali-quantitativo riguardanti le piastrine e la sintesi delle proteine implicate nella cascata della coagulazione (50).

Allo stesso tempo, la CKD risulta essere un determinante essenziale per la genesi di fenomeni pro-trombotici grazie al disequilibrio tra fattori favorenti e fattori protettivi la formazione del trombo (aumento dei livelli plasmatici di fibrinogeno, diminuzione dell'attività fibrinolitica e iperaggregabilità piastrinica) (50).

Ritenzione di tossine uremiche, anemia ed alterazioni del metabolismo dell'ossido nitrico sono solo alcuni dei meccanismi fisiopatologici alla base della disfunzione piastrinica (51).

Nei pazienti affetti da CKD ed ESRD sono stati documentati sia un'inadeguata risposta all'aggregazione piastrinica indotta da stimoli diversi (ADP, per esempio) sia un aumento dell'aggregabilità piastrinica (50).

Evidenze di tipo indiretto suggeriscono come lo stato di ipercoagulabilità dei pazienti sottoposti a trattamento emodialitico sia associato a diverse anomalie di tipo biochimico: aumento dei livelli sierici di fibrinogeno, aumentata attività di alcuni dei fattori della coagulazione (VII, VIII, fattore di Von Willebrand, fattore tissutale) e diminuita attività di altri (antitrombina III, proteine C e S, fattori II, IX, X e XII), nonostante le loro concentrazioni plasmatiche si presentino spesso normali o persino aumentate $(50,52)$.

Nei pazienti sottoposti a dialisi peritoneale è documentabile un particolare profilo per quanto riguarda l'assetto della coagulazione: aumento dei livelli plasmatici di fibrinogeno, aumento dell'attività dei fattori II, VII, VIII, IX, X e XII, elevate concentrazioni plasmatiche di proteina $S$ e concentrazioni normali di antitrombina III e proteina C (53-55).

Nei pazienti sottoposti a trattamento emodialitico sono stati evidenziati sia un aumento della fibrinolisi (probabilmente secondaria all'attivazione della cascata della coagulazione) sia un deficit della stessa attività fibrinolitica (56).

Per tutte le motivazioni di ordine fisiopatologico elencate sopra, valutare il potenziale rapporto rischio/beneficio del trattamento anticoagulante nei pazienti con ESRD e FA non valvolare rimane un esercizio sostanzialmente complicato.

Le Linee Guida KDIGO (Kidney Diseases Improving Global Outcomes) non raccomandano l'impiego del warfarin per la prevenzione dello stroke nei pazienti dializzati affetti da FA non valvolare (8).

Uno studio di Chen et al., effettuato su una coorte di 134.000 pazienti affetti da ESRD (eGFR $<15 \mathrm{~mL} / \mathrm{min}$ ), ha evidenziato come l'impiego di warfarin o di farmaci antiaggreganti non comporti la riduzione dell'incidenza di ictus ischemico nei pazienti con ESRD (57). Allo stesso tempo, in una popolazione di 1626 pazienti sottoposti a trattamento dialitico (emodialisi o dialisi peritoneale), il trattamento con warfarin non solo non ha consentito di ridurre il rischio di stroke ischemico ma è stato associato a un incremento del rischio emorragico (58).

Evidenze opposte sono quelle risultanti da uno studio cinese condotto su pazienti affetti da FA non valvolare con normofunzione renale, pazienti in trattamento mediante dialisi peritoneale (DP) e pazienti affetti da CKD ma con rischio di stroke ischemico paragonabile ai soggetti in trattamento mediante DP: nello studio in oggetto, il trattamento con warfarin 
era associato a una riduzione del rischio di stroke ischemico in assenza di un aumento del rischio emorragico (59).

Nello studio di coorte svedese sulla FA comprendente 307.000 pazienti affetti da $F A$, dei quali 13.000 presentavano una pregressa diagnosi di insufficienza renale, la maggior parte dei pazienti ha tratto beneficio dalla terapia con warfarin nonostante l'elevato rischio emorragico (60).

Come già anticipato, sebbene la FDA non abbia formalmente approvato l'impiego di apixaban in pazienti con eGFR $<15 \mathrm{~mL} / \mathrm{min}$, ne raccomanda l'impiego nei pazienti in trattamento emodialitico anche se solo sulla base delle proprietà farmacocinetiche e non di eventuali risultanze di trial clinici (61).

In accordo con le Linee Guida AHA (American Heart Association), ACC (American College of Cardiology) e HRS (Heart Rhythm Society), risulta ragionevole prescrivere warfarin in pazienti con un $\mathrm{CHA}_{2} \mathrm{DS}_{2}$-VASc score $\geq 2$ ed ESRD (eGFR $<15 \mathrm{~mL} / \mathrm{min}$ ) (62).

Allo stato attuale, il trattamento anticoagulante nei pazienti affetti da FA ed ESRD rimane un problema non completamente risolto e tale dilemma non si risolverà almeno fino agli eventuali risultati di trial clinici dedicati (63).

I NOAC, come da Linee Guida ESC 2016, non sono attualmente raccomandati nei pazienti affetti da ESRD (eGFR $<15 \mathrm{~mL} / \mathrm{min}$ ) o in trattamento sostitutivo dialitico a causa della mancanza di evidenze in termini di rapporto rischio/ beneficio e di trial clinici dedicati (62).

Alla luce delle Linee Guida EHRA ed ESC, i NOAC possono essere considerati sicuri ed efficaci, rispetto al warfarin, in pazienti con eGFR compreso tra 30 e $49 \mathrm{~mL} / \mathrm{min}(64,65)$.
Nei pazienti con eGFR $<15 \mathrm{~mL} / \mathrm{min}$, il warfarin va considerato di prima scelta rispetto ai NOAC almeno fino a che non si avranno risultati derivanti da trial clinici randomizzati e controllati.

Nei pazienti con eGFR compreso tra 30 e $49 \mathrm{~mL} / \mathrm{min}$, la prima scelta terapeutica deve essere individuata nei NOAC e, più in particolare, in apixaban $5 \mathrm{mg}$ BID (o $2.5 \mathrm{mg} \mathrm{BID}$ nel caso in cui vi siano due dei seguenti tre criteri: età $\geq 80$ anni, peso corporeo $\leq 60 \mathrm{~kg}$, creatinina sierica $\geq 1.5 \mathrm{mg} / \mathrm{dL}$ ), rivaroxaban $15 \mathrm{mg} /$ die o edoxaban $30 \mathrm{mg} /$ die.

Una terapia di seconda scelta può essere individuata in dabigatran al dosaggio di $110 \mathrm{mg}$ BID, mentre vanno evitati dabigatran al dosaggio di $150 \mathrm{mg}$ BID, rivaroxaban al dosaggio di $20 \mathrm{mg} /$ die ed edoxaban al dosaggio di $60 \mathrm{mg} /$ die (Tab. II).

Come già anticipato, non vi sono dati clinici significativi prodotti da trial randomizzati per i pazienti sottoposti a trattamento dialitico, sebbene la FDA abbia approvato l'impiego di apixaban in pazienti emodializzati (al dosaggio di $2.5 \mathrm{mg}$ BID) pur in assenza di dati riguardanti la sicurezza del trattamento. Pertanto, in questa popolazione di pazienti, il trattamento di scelta resta quello con warfarin (66).

Per quanto concerne alcuni trial clinici in via di definizione, va menzionato lo studio XARENO (Factor XA - Inhibition in RENal Patients With Non-valvular Atrial Fibrillation - Observational Registry), che ha l'obiettivo di valutare l'impatto sugli outcome clinici della terapia con Rivaroxaban $15 \mathrm{mg} /$ die in pazienti con eGFR compreso tra 15 e $49 \mathrm{~mL} / \mathrm{min}$ (67).

Sono, inoltre, da poco disponibili i dati di uno studio condotto su 16 pazienti affetti da ESRD in trattamento con

TABELLA II - Linee guida European Society of Cardiology (ESC) per l'impiego dei NOAC in pazienti con malattia renale cronica (CKD) e fibrillazione atriale (FA) non valvolare

\section{eGFR $15-49 \mathrm{~mL} / \mathrm{min}$}

Prima scelta
Apixaban 5 mg BID (o 2.5 mg BID in presenza di due dei seguenti tre criteri: età $\geq 80$ anni, peso corporeo $\leq 60 \mathrm{~kg}$, creatinina sierica $\geq 1.5 \mathrm{mg} / \mathrm{dL}$ )

Rivaroxaban $15 \mathrm{mg} /$ die

Edoxaban $30 \mathrm{mg} /$ die

Seconda scelta

Dabigatran 110 mg BID

Non raccomandato
Dabigatran 150 mg BID

Rivaroxaban $20 \mathrm{mg} /$ die

Edoxaban $60 \mathrm{mg} /$ die
eGFR $<15 \mathrm{~mL} / \mathrm{min}$

Prima scelta

Non raccomandato
Nessuna terapia anticoagulante (warfarin)

Dabigatran, rivaroxaban, apixaban*, edoxaban

*FDA ha approvato (solo in USA) l'impiego di apixaban in pazienti emodializzati

eGFR $>95 \mathrm{~mL} / \mathrm{min}$

Prima scelta

Dabigatran $150 \mathrm{BID}$, rivaroxaban $20 \mathrm{mg} /$ die, apixaban $5 \mathrm{mg}$ BID

Non raccomandato

Edoxaban $60 \mathrm{mg} /$ die 
Rivaroxaban $15 \mathrm{mg} /$ die. Gli Autori, in questo caso, concludono affermando come il profilo farmacocinetico e farmacodinamico della molecola non risulti particolarmente modificato nei pazienti con ESRD (68).

Apixaban è stato testato in pazienti sottoposti a trattamento emodialitico (trial NCT02672709) al dosaggio di $2.5 \mathrm{mg}$ BID; al momento è terminata la fase di arruolamento e si è in attesa dei risultati finali (69).

In un altro studio, condotto su pazienti affetti da ESRD, Apixaban si è rivelato superiore al warfarin per quanto concerne il rischio di sanguinamenti maggiori e il rischio composito (sanguinamenti maggiori e minori), mentre si è osservata una sostanziale parità per quanto concerne il rischio di stroke (70).

Anche Edoxaban, testato in una piccola coorte di pazienti, non ha evidenziato un profilo di rischio superiore nei pazienti sottoposti a trattamento emodialitico, in quanto le concentrazioni del farmaco (impiegato al dosaggio di $15 \mathrm{mg} / \mathrm{die}$ ) non differivano significativamente tra periodo immediatamente post-dialitico e intervallo interdialitico (71).

\section{Conclusioni}

In conclusione è possibile affermare come la FA rappresenti di gran lunga l'aritmia più frequente nei pazienti affetti da CKD ed ESRD.

La prescrizione di una terapia anticoagulante in questa tipologia di pazienti si presenta particolarmente difficoltosa alla luce del profilo farmacologico dei NOAC e delle caratteristiche, non sempre favorevoli, del warfarin.

Pur con i suoi limiti, quest'ultimo deve rappresentare tuttora la terapia di scelta nei pazienti affetti da ESRD (eGFR $<15 \mathrm{~mL} / \mathrm{min}$ ), rimandando l'impiego dei NOAC alla popolazione di soggetti con eGFR $>15 \mathrm{~mL} / \mathrm{min}$.

\section{Disclosures}

Financial support: No financial support was received for this submission.

Conflict of interest: The authors have no conflict of interest.

\section{Bibliografia}

1. Camm J, Lip GY, Caterina R, et al. ESC Committee for Practice Guidelines (CPG). 2012 focused update of the ESC Guidelines for the management of atrial fibrillation. Eur Heart J. 2012; 33:2719-2747.

2. Camm J, Kirchhof P, Lip GY, et al. Guidelines for the management of atrial fibrillation. Eur Heart J. 2010;31:2369-2429.

3. Zoni-Berisso M, Lercari F, Carazza T, Domenicucci SI. Epidemiology of atrial fibrillation: European perspective. Clin Epidemiol. 2014;6:213-220.

4. Naghavi $M$, Wang $H$, Lozano $R$, et al. Global, regional, and national age-sex specific all-cause and cause-specific mortality for 240 causes of death, 1990-2013: A systematic analysis for the Global Burden of Disease Study. Lancet. 2013; 385:117-171.

5. Herzog CA, Asinger RW, Berger AK, et al. Cardiovascular disease in chronic kidney disease. A clinical update from
Kidney Disease: Improving Global Outcomes (KDIGO). Kidney Int. 2011;80:572-586.

6. Shiroshita-Takeshita A, Brundel BJ, Nattel S. Atrial fibrillation: basic mechanisms, remodeling and triggers. J Interv Card Electrophysiol. 2005;13:181-193.

7. Wetmore JB, Mahnken JD, Rigler SK, et al. The prevalence of and factors associated with chronic atrial fibrillation in Medicare/Medicaid-eligible dialysis patients. Kidney Int. 2012; 81:469-476.

8. Genovesi S, Pogliani D, Faini A, et al. Prevalence of atrial fibrillation and associated factors in a population of long-term hemodialysis patients. Am J Kidney Dis. 2005;46:897-902.

9. Soliman EZ, Prineas RJ, Go AS, et al. Chronic kidney disease and prevalent atrial fibrillation: the Chronic Renal Insufficiency Cohort (CRIC). Am Heart J. 2010;159:1102-1107.

10. Alonso A, Lopez FL, Matsushita K, et al. Chronic kidney disease is associated with the incidence of atrial fibrillation: the Atherosclerosis Risk in Communities (ARIC) Study. Circulation. 2011;123:2946-2953.

11. Baber U, Howard VJ, Halperin JL, et al. Association of chronic kidney disease with atrial fibrillation among adults in the United States: Reasons for geographic and racial differences in stroke (REGARDS) study. Circ Arrhythm Electrophysiol. 2011;4:26-32.

12. Wizemann $\mathrm{V}$, Tong $\mathrm{L}$, Satayathum $\mathrm{S}$, et al. Atrial fibrillation in hemodialysis patients: clinical features and associations with anticoagulant therapy. Kidney Int. 2010;77:1098-1106.

13. Fujii $\mathrm{H}, \mathrm{Kim} \mathrm{JI}$, Yoshiya $\mathrm{K}$, et al. Clinical characteristics and cardiovascular outcomes of hemodialysis patients with atrial fibrillation: a prospective follow-up study. Am J Nephrol. 2011; 34:126-134.

14. Vázquez-Ruiz de Castroviejoa E, Sánchez-Perales C, Lozano Cabezas $\mathrm{C}$, et al. Incidence of atrial fibrillation in hemodialysis patients: a prospective long-term follow-up study. Rev Esp Cardiol. 2006;59:779-784.

15. Genovesi S, Vincenti A, Rossi E, et al. Atrial fibrillation and morbidity and mortality in a cohort of long-term hemodialysis patients. Am J Kidney Dis. 2008;51:255-262.

16. Vazquez E, Sanchez-Perales C, Garcia-Garcia F, et al. Atrial fibrillation in ncident dialysis patients. Kidney Int. 2009;76: 324-330.

17. Goldstein BA, Arce CM, Hlatky MA, et al. Trends in the incidence of atrial fibrillation in older patients initiating dialysis in the United States. Circulation. 2012;126:2293-2301.

18. Grande A. Atrial fibrillation and dialysis. A convergence of risk factors. Rev Esp Cardiol. 2006;59:766-769.

19. Korantzopoulos P, Liu T, Li L, et al. Implantable cardioverter defibrillator therapy in chronic kidney disease: a meta-analysis. Europace. 2009;11:1469-1475.

20. Xu D, Murakoshi N, Sairenchi T, et al. Anemia and reduced kidney function as risk factors for new onset of atrial fibrillation (from the Ibaraki prefectural health study). Am J Cardiol. 2015;115:328-333.

21. Hirakata $H$, Tsubakihara $Y$, Gejyo $F$, et al. Maintaining high hemoglobin levels improved the left ventricular mass index and quality of life scores in pre-dialysis Japanese chronic kidney disease patients. Clin Exp Nephrol. 2010;14:28-35.

22. Friberg L, Benson L, Lip GY. Balancing stroke and bleeding risks in patients with atrial fibrillation and renal failure: the Swedish Atrial Fibrillation Cohort study. Eur Heart J. 2015;36:297-306.

23. Reinecke $H$, Brand $E$, Mesters $R$, et al. Dilemmas in the management of atrial fibrillation in chronic kidney disease. J Am Soc Nephrol. 2009;20:705-711.

24. Go AS, Fang MC, Udaltsova N, et al. Impact of proteinuria and glomerular filtration rate on risk of thromboembolism in atrial 
fibrillation: the Anti-coagulation and Risk Factors in Atrial Fibrillation (ATRIA) study. Circulation. 2009;119:1363-1369.

25. Olesen JB, Lip GY, Kamper AL, et al. Stroke and bleeding in atrial fibrillation with chronic kidney disease. N Engl J Med. 2012;367:625-635.

26. Lip GY. Chronic renal disease and stroke in atrial fibrillation: balancing the prevention of thromboembolism and bleeding risk. Europace. 2011;13:145-148.

27. Mitsuma W, Matsubara T, Hatada K, et al. Clinical characteristics of hemodialysis patients with atrial fibrillation: The RAKUEN (Registry of atrial fi- brillation in chronic kidney disease under hemodialysis from Niigata) study. J Cardiol. 2016;68(2):148-155.

28. Mancuso, P, Antoniotti, P, Quarna, J, et al. Validation of a standardized method for enumerating circulating endothelial cells and progenitors: flow cytometry and molecular and ultrastructural analyses. Clin Cancer Res. 2009;15:267-273.

29. Malyszko J. Mechanism of endothelial dysfunction in chronic kidney disease. Clin Chim Acta. 2010;411:1412-1420.

30. Zeng WT, Sun XT, Tang K, et al. Risk of thromboembolic events in atrial fibrillation with chronic kidney disease. Stroke. 2015;46:157-163.

31. Hohnloser SH. Stroke prevention versus bleeding risk in atrial fibrillation: a clinical dilemma. J Am Coll Cardiol. 2011;57: 181-183.

32. Connolly SJ, Ezekowitz MD, Yusuf S, et al. RE-LY Steering Committee and Investigators. Dabigatran versus warfarin in patients with atrial fibrillation. N Engl J Med. 2009;361: 1139-1151.

33. Chatrou ML, Winckers K, Hackeng TM, et al. Vascular calcification: the price to pay for anticoagulation therapy with vitamin K-antagonists. Blood Rev. 2012;26:155-166.

34. Brodsky SV, Nadasdy T, Rovin BH, et al. Warfarin-related nephropathy occurs in patients with and without chronic kidney disease and is associated with an increased mortality rate. Kidney Int. 2011;80:181-189.

35. Patel MR, Mahaffey KW, Garg J, et al. for the ROCKET AF Steering Committee. Rivaroxaban versus warfarin in nonvalvular atrial fibrillation. N Engl J Med 2011;365:883-891.

36. Heidbuchel $H$, Verhamme $P$, Alings $M$, et al. Updated European Heart Rhythm Association practical guide on the use of nonvitamin $\mathrm{K}$ antagonist anticoagulants in patients with nonvalvular atrial fibrillation. Europace. 2015;17:1467-1507.

37. Stangier J, Rathgen $\mathrm{K}$, Stähle $\mathrm{H}$, et al. Influence of renal impairment on the pharmacokinetics and pharmacodynamics of oral dabigatran etexilate: an open-label, parallel-group, single-centre study. Clin Pharmacokinet. 2010;49:259-268.

38. Kubitza D, Becka M, Mueck W, et al. Effects of renal impairment on the pharmacokinetics, pharmacodynamics and safety of rivaroxaban, an oral, direct factor $\mathrm{Xa}$ inhibitor. $\mathrm{Br} \mathrm{J}$ Clin Pharmacol. 2010;70:703-712.

39. Granger $\mathrm{CB}$, Alexander JH, McMurray JJV, et al. for the ARISTOTLE Committees and Investigators. Apixaban versus warfarin in patients with atrial fibrillation. $\mathrm{N}$ Engl J Med. 2011;365:981-992.

40. Fox KAA, Piccini JP, Wojdyla D, et al. Prevention of stroke and systemic embolism with rivaroxaban compared with warfarin in patients with non-valvular atrial fibrillation and moderate renal impairment. Eur Heart J. 2011;32:2387-2394.

41. De Vriese AS, Caluwé R, Bailleul E, et al. Dose-finding study of rivaroxaban in hemodialysis pa- tients. Am J Kidney Dis. 2015;66:91-98.

42. Coppens M, Synhorst D, Eikelboom JW, et al. Efficacy and safety of apixaban compared with aspirin in patients who previously tried but failed treatment with vitamin $\mathrm{K}$ antagonists: results from the AVERROES trial. Eur Heart J. 2014;35:1856-1863.

43. Levey AS, Stevens LA, Schmid CH, et al. for the CKD-EPI (Chronic Kidney Disease Epidemiology Collaboration). A new equation to estimate glomerular filtration rate. Ann Intern Med. 2009;150:604-612.

44. Parasrampuria DA, Truitt KE. Pharmacokinetics and pharmacodynamics of edoxaban, a non- vitamin $k$ antagonist oral anticoagulant that inhibits clotting factor Xa. Clin Pharmacokinet. 2016;55:641-655.

45. Bohula EA, Giugliano RP, Ruff $C T$, et al. The impact of renal function on outcomes with edoxaban in the ENGAGE AF-TIMI Trial. Circulation. 2015;132:A17169.

46. Daiichi-Sankyo, Inc. Savaysa (endoxaban) NDA 206316. FDA Advisory Committee briefing document. Available at: http://www.fda.gov/downloads/advisorycommittees/ committeesmeeting materials/drugs/cardiovascularandrenaldrugsadvisory committee/ucm420705.pdf. Accessed April 7, 2016.

47. Koretsune $Y$, Yamashita T, Kimura T, et al. Short-term safety and plasma concentrations of edoxaban in Japanese patients with non-valvular atrial fibrillation and severe renal impairment. Circ J. 2015;79:1486-1495.

48. Parasrampuria DA, Marbury T, Matsushima $N$, et al. Pharmacokinetics, safety, and tolerability of edoxaban in end-stage renal disease subjects undergoing haemodialysis. Thromb Haemost. 2015;113:719-727.

49. Eisenhower C, Koronkowski M, Marcum Z. Implications of Recent Drug Approvals for Older Adults. Ann Longterm Care. 2016;24(1):25-30.

50. Małyszko J, Małyszko JS, Myśliwiec M, Buczko W. Hemostasis in chronic renal failure. Rocz Akad Med Bialymst. 2005;50: 126-131.

51. Rabelink TJ, Zwaginga JJ, Koomans HA, Sixma JJ. Thrombosis and hemostasis in renal disease. Kidney Int. 1994;46:287-296.

52. Malyszko J, Malyszko JS, Kozminski P, et al. Adipokines, linking adipocytes and vascular function in hemodialyzed patients, may also be possibly related to CD146, a novel adhesion molecule. Clin Appl Thromb Hemostas. 2008;14:338-345.

53. Małyszko J, Małyszko JS, Mysliwiec M. Comparison of hemostasis between CAPD and HD patients. Perit Dial Int. 2001;21:158-165.

54. Małyszko J, Małyszko JS, Myśliwiec M. Endothelial cell injury markers in chronic renal failure on conservative treatment and continuous ambulatory peritoneal dialysis (CAPD). Kidney Blood Press Res. 2004;27:71-77.

55. Malyszko J, Malyszko JS, Wolczynski S, et al. Adiponectin is related to CD146, a novel marker of endothelial cell activation/ injury in chronic renal failure and peritoneally dialyzed patients. J Clin Endocrinol Metab. 2004;89:4620-4627.

56. Adams MJ, Irish $A B$, Watts $G F$, et al. Hypercoagulability in chronic kidney disease is associated with coagulation activation but not endothelial function. Thromb Res. 2008;123:374-380.

57. Chen JJ, Lin LY, Yang YH, et al. Anti-platelet or anti-coagulant agent for the prevention of ischemic stroke in patients with end-stage renal disease and atrial fibrillation-a nation-wide database analyses. Int J Cardiol. 2014;177:1008-1011.

58. Shah M, Avgil Tsadok M, Jackevicius CA, et al. Warfarin use and the risk for stroke and bleeding in patients with atrial fibrillation undergoing dialysis. Circulation. 2014;129:1196-1203.

59. Chan PH, Huang D, Yip PS, et al. Ischaemic stroke in patients with atrial fibrillation with chronic kidney disease undergoing peritoneal dialysis. Europace. 2016;18(5):665-671.

60. Friberg L, Benson L, Lip GY. Balancing stroke and bleeding risks in pa- tients with atrial fibrillation and renal failure: the Swedish Atrial Fibrillation Cohort study. Eur Heart J. 2015;36:297-306. 
61. Heidbuchel $H$, Verhamme $P$, Alings $M$, et al. Updated European Heart Rhythm Association Practical Guide on the use of nonvitamin $\mathrm{K}$ antagonist anticoagulants in patients with nonvalvular atrial fibrillation. Europace. 2015;17:1467-1507.

62. January CT, Wann LS, Alpert JS, et al. 2014 AHA/ACC/ HRS guideline for the management of patients with atrial fibrillation: executive summary: a report of the American College of Cardiology/American Heart Association Task Force on practice guidelines and the Heart Rhythm Society. Circulation. 2014;130:2071-2104.

63. Schwartzenberg S, Lev El, Sagie A, et al. The quandary of oral anticoagulation in patients with atrial fibrillation and chronic kidney disease. Am J Cardiol. 2016;117:477-482.

64. Fox KA, Piccini JP, Wojdyla D, et al. Prevention of stroke and systemic embolism with rivaroxaban compared with warfarin in patients with non-valvular atrial fibrillation and moderate renal impairment. Eur Heart J. 2011;32:2387-2394.

65. Hijazi Z, Hohnloser SH, Oldgren J, et al. Efficacy and safety of dabigatran compared with warfarin in relation to baseline renal function in patients with atrial fibrillation: A RE-LY (Randomized
Evaluation of Long-term Anticoagulation Therapy) trial analysis. Circulation. 2014;129:961-970.

66. Chan KE, Lazarus JM, Thadhani R, et al. Warfarin use associates with increased risk for stroke in hemodialysis patients with atrial fibrillation. J Am Soc Nephrol. 2009;20:2223-2233.

67. https://clinicaltrials.gov/ct2/show/NCT02663076?term=XARE NO\&rank=1).

68. Dias C, Moore KT, Murphy J, Ariyawansa J, Smith W, Mills RM, Weir MR. Pharmacokinetics, pharmacodynamics and safety of single-dose rivaroxaban in chronic hemodialysis. Am J Nephrol. 2016;43:229-236

69. https://clinicaltrials.gov/ct2/show/NCT02672709.

70. Stanton BE, Barasch NS, Tellor KB. Comparison of the safety and effectiveness of apixaban versus warfarin in patients with severe renal impairment. Pharmacotherapy. 2017;37(4): 412-419.

71. Parasrampuria DA, Marbury $\mathrm{T}$, Matsushima $\mathrm{N}$, et al. Pharmacokinetics, safety, and tolerability of edoxaban in endstage renal disease subjects undergoing hemodialysis. Thromb and Haemost. 2017;117(4):821. 